\title{
Probing the Irradiation Defects in Enhanced 2G High Temperature Superconducting Wire
}

\author{
Yi Zhang ${ }^{1}$, M. W. Rupich ${ }^{2}$, Vyacheslav Solovyov ${ }^{3}$, Qiang Li $^{4}$ and Amit Goyal ${ }^{\text {* }}$ \\ 1. Research and Education in Energy, Environment \& Water (RENEW), State University of New York \\ (SUNY), Buffalo, NY, USA. \\ 2. AMSC, MA, USA. \\ 3. Brookhaven Technology Group, Stony Brook, NY, USA. \\ 4. Brookhaven National Laboratory, Upton, NY, USA. \\ * Corresponding author: agoyal@buffalo.edu
}

Significant efforts have been made for decades to enhance the functionalities of HTS rare earth barium copper oxide $\mathrm{REBa}_{2} \mathrm{Cu}_{3} \mathrm{O}_{7-\delta}$ (REBCO) by introducing artificial vortex pinning microstructures. A variety of defects in HTS composites have been proven to serve as effective pinning centers [1]. Past research has shown that particle irradiation is an effective approach to incorporate highly engineered defects structures into superconductor matrix [2-4]. Irradiation with high energy heavy ions generates columnar defects, whereas irradiation with electrons, protons, neutrons, and light ions produces atomicscale or nano-scale defects [5]. By choosing appropriate mass and energy of irradiating species, we are can manipulate the density and morphology of artificial irradiation induced defects. The defects are generated by the depletion and disorder of $\mathrm{O}$ and $\mathrm{Cu}$ around the areas where collision happens between irradiation species and YBCO. Introducing artificial irradiation defects can achieve significant enhancement in critical current density $J_{c}$ by producing strong, uniformly distributed pinning centers.

In this work, we present a detailed investigation of the atomic and chemistry structures of irradiation defects in AMSC's second generation (2G) HTS coil wire by using aberration-corrected scanning transmission electron microscopy (AC-STEM) coupled with atomic resolution electron energy loss spectroscopy (EELS). A 1.2 $\mu \mathrm{m}$ thick Dy-doped YBCO films was deposited onto Ni(5at\%)W RABiTS substrate by metal organic deposition (MOD) process [6]. The ion irradiation was carried out at Brookhaven National Laboratory using the tandem Van de Graaff accelerators with 18-MeV $\mathrm{Au}^{5+}$ ions to a dose of $2 \times 10^{11} \mathrm{Au} / \mathrm{cm}^{2}$ along the c-axis of YBCO film. Prior to the irradiation, the coil wire sample has a $77 \mathrm{~K}$, self-field $I_{c}$ of around $385 \mathrm{~A} / \mathrm{cm}-\mathrm{w}$. In comparison, the value decreased to $320 \mathrm{~A} / \mathrm{cm}-\mathrm{w}$ after irradiation.

The irradiation defects in YBCO film are amorphous nanoclusters with the size of around 2-5 $\mathrm{nm}$. The amorphous nanoclusters were surrounded by highly distorted YBCO layers. The EELS map with a spatial step size of $0.24 \mathrm{~nm}$ was acquired includes both defects and surrounding matrix (Figure 1a). The variation of $\mathrm{Cu}$ and $\mathrm{O}$ relative to $\mathrm{Ba}$ were extracted from the area across the defect (Figure 1b). Quantitative analysis suggests that the $\mathrm{O} / \mathrm{Ba}$ ratio varies from 3 in matrix to $\sim 1.5$ in defect region, which shows high concentration of oxygen deficiency. The $\mathrm{Cu}-L_{2,3}$ edge fine structure extracted from defect, adjacent matrix, and distant matrix areas were plotted in Figure 1c. $\mathrm{Cu} L_{3}$ and $L_{2}$ white lines are very sensitive to the change in valence states. Both the spectra from adjacent and distant matrix areas show splitting peaks at $934.5 \mathrm{eV}$ (peak $a$ ) and $937.2 \mathrm{eV}$ (peak $b$ ), resulting from the existence of mixed valence states of $\mathrm{Cu}^{2+}$ (peak $a$ ) and $\mathrm{Cu}^{1+}$ (peak $b$ ) in YBCO matrix. It is worth noting that the spectra from the defect area only shows peak $b$. The diminished peak $a$ indicates that the $\mathrm{Au}^{5+}$ ion irradiation caused oxygen deficiency defects transforming $\mathrm{Cu}$ in $\mathrm{YBCO}$ structure from $\mathrm{Cu}^{2+}$ to $\mathrm{Cu}^{1+}$. 
Our results showed that irradiation of the YBCO wire with $18 \mathrm{MeV} \mathrm{Au}^{5+}$ ions produced highly oxygen deficient amorphous nanoclusters. Compared to the non-irradiated sample, the irradiated sample showed a significant decrease in $T_{c}$ and self-field $I_{c}$. A more detailed study shows that the degradation is related to the nanoscale defects and point oxygen vacancy defects in YBCO structure [7].

\section{References:}

[1] SR Foltyn et al., Nature Materials 6 (2007), p. 631.

[2] RB Van Dover et al., Nature 342 (1989), p. 55.

[3] L Civale et al., Physical Review Letters 65 (1990), p. 1164.

[4] FM Sauerzopf et al., Physical Review B 43 (1991), p. 3091.

[5] W-K Kwok et al., Rep. Prog. Phys. 79 (2016), p. 116501.

[6] MW Rupich et al., IEEE Transactions on Applied Superconductivity 26 (2016), p. 1.

[7] The authors acknowledge funding from the DOE/EERE under contract No: DE-EE0007870.

The authors acknowledge support from U.S. Department of Energy Office of Energy Efficiency \& Renewable Energy, Advanced Manufacturing Office for this program.

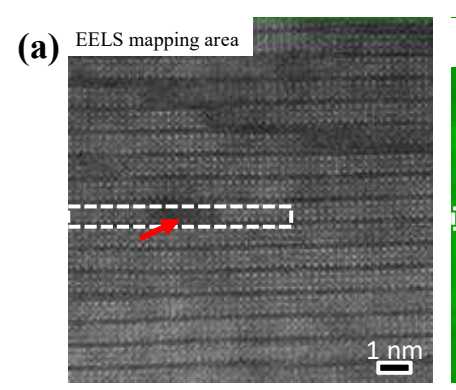

(b)

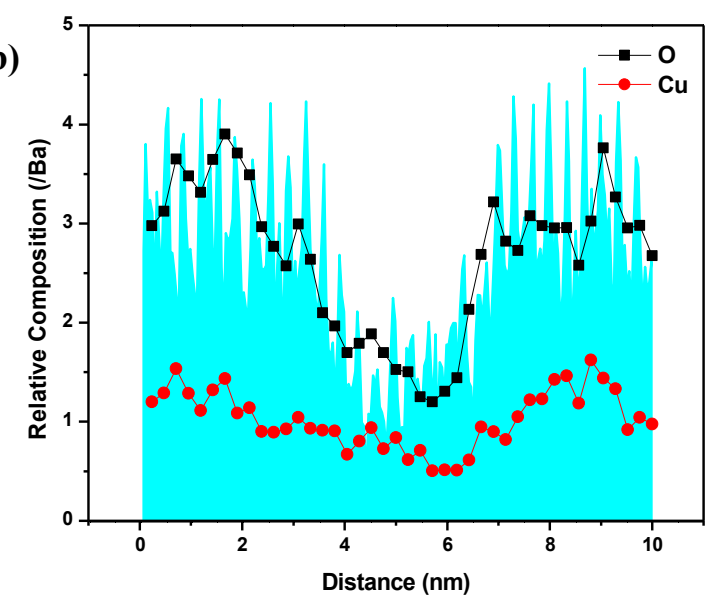

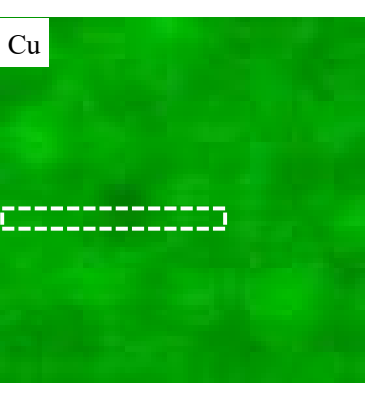

(c)
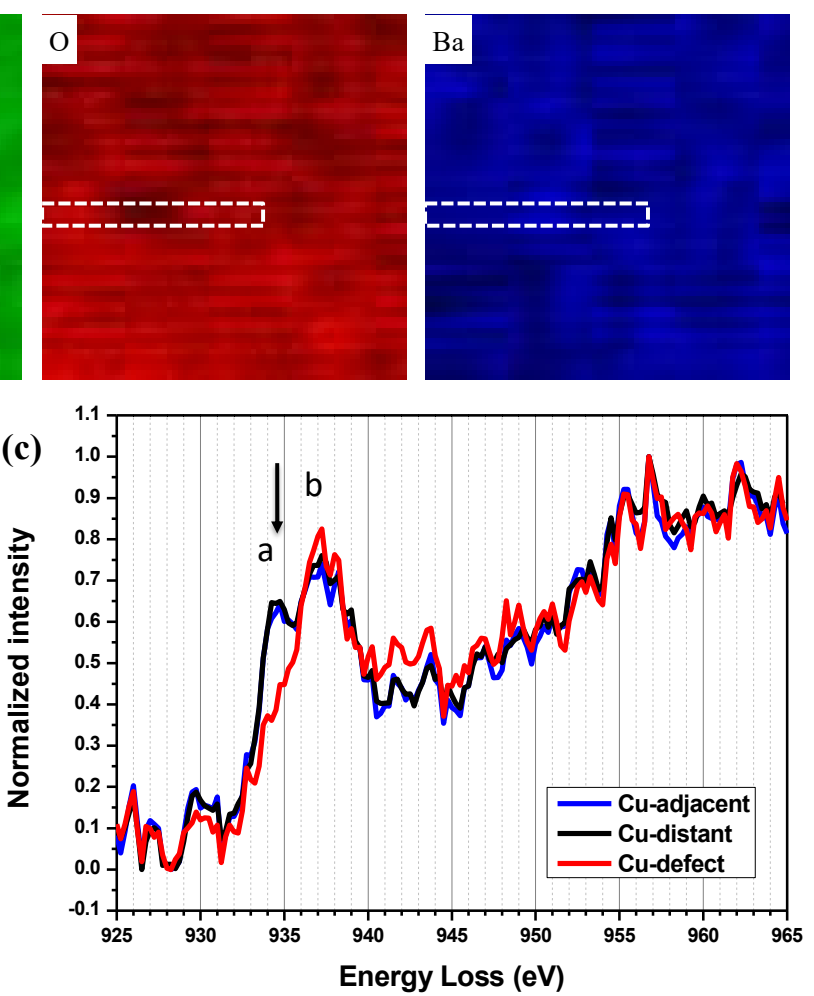

Figure 1. Two-dimensional EELS map was acquired from irradiated film. (a) The area includes both defect and surrounding YBCO matrix. (b) $\mathrm{Cu}, \mathrm{O}$ relative to $\mathrm{Ba}$ were extracted from the dashed area. The background is the HAADF intensity profile from the same area which includes $1 \times 29$ unit cells. A dramatically lower $\mathrm{O} / \mathrm{Ba}$ ratio indicates that the irradiation defect region is highly oxygen-deficient. (c) The $\mathrm{Cu}-L_{2,3}$ edge fine structures are extracted from the marked defect, adjacent matrix, and distant matrix areas. The diminished peak $a$ indicates oxygen deficiency transforming $\mathrm{Cu}$ from $\mathrm{Cu}^{2+}$ to $\mathrm{Cu}^{1+}$. 